\title{
Developmental Expression of Defensive Responses During Exposure to Conspecific Adults in Preweanling Rats (Rattus norvegicus)
}

\author{
Lorey K. Takahashi \\ Department of Psychiatry, University of Wisconsin Medical School \\ and William S. Middleton Memorial Veterans Hospital, Madison, Wisconsin
}

\begin{abstract}
I examined preweanling rats' (Rattus norvegicus) expression of ultrasounds and secretion of ACTH when exposed to unfamiliar adult male rats or to their mothers. Pups at 7 days of age produced similar levels of ultrasonic vocalization near both unfamiliar males and mothers. However, these pups could discriminate familiar from unfamiliar adults because ACTH was significantly higher in pups near adult males than in those near mothers. At 14 days of age, pups avoided adult males but not their mothers; therefore, adult males represented a significant threat. Importantly, 14-day-old rats significantly reduced ultrasound production only when near adult males. Pups at 21 days of age no longer emitted ultrasounds when socially isolated or when near conspecific adults. In addition, 14- and 21-day-old rats produced similar elevated ACTH levels across stimulus conditions. Results show significant changes in preweanling rats' responses to conspecific adults.
\end{abstract}

After a gestation period of $22-23$ days, the Norway rat delivers a litter of altricial young (Rosenblatt \& Lehrman, 1963). These initially helpless rat pups mature rapidly and are able to survive independently of the mother after 3 weeks. The ability to locomote or walk develops toward the end of the 2nd week of life (Altman \& Sudarshan, 1975), and at this time, pups begin to crawl out of the nest (Bolles \& Woods, 1964; Rosenblatt \& Lehrman, 1963). In addition, the eyes begin to open, and intake of solid foods commences shortly thereafter (Bolles \& Woods, 1964). Obviously, if the young rat is to survive to reproductive age, it must respond appropriately to the increasing number of threatening stimuli encountered outside the nest. I examined preweanling rats' ability to modulate some components of their defensive repertoire during this 3-week period of rapid maturational growth.

In a number of altricial preweanling rodents, ultrasonic vocalizations are frequently exhibited when the pup is perturbed, and these vocalizations are interpreted to reflect a state of distress (De Ghett, 1974; Hart \& King, 1966; Noirot, 1966, 1968; Zippelius \& Schleidt, 1956). The elicitation of these ultrasounds can be produced by removing the pup from the nest and placing it in social isolation. Studies have reported that under these circumstances the emission of ultrasounds disappears gradually by the 3rd week of life (Noirot, 1972; Takahashi, Baker, \& Kalin, 1990). The emission of ultrasounds by preweanling pups may serve to solicit maternal attention and their retrieval to the nest (Allin \& Banks, 1972; Noirot, 1972; Smotherman, Bell, Starzec, Elias, \& Zachman, 1974).

The research was supported by National Institutes of Health Grant MH-43986.

I thank C. Haglin and $J$. Turner for technical assistance.

Correspondence concerning this article should be addressed to Lorey K. Takahashi, Department of Psychiatry, University of Wisconsin Medical School, 600 Highland Avenue, Madison, Wisconsin 53792.
Recently, colleagues and I (Takahashi, Baker, \& Kalin, 1990 ) demonstrated that 14-day-old rats tested in social isolation varied their production of ultrasonic vocalizations after they were administered electric foot shock. Rats that were tested in social isolation and shocked produced fewer ultrasounds than ones only tested in social isolation. The results suggested that rats develop by 14 days of age the capacity to inhibit or modulate their emission of ultrasounds in response to intense stimulation. This ability of 14-day-old rats to cease production of ultrasounds and remain inconspicuous may facilitate survival at a time when they may be highly vulnerable to predators or hostile conspecific adults that are encountered outside the nest.

In order to further document the emergence of this ability of 2-week-old rats to reduce their production of ultrasounds when exposed to stressful stimuli, I conducted studies in which preweanling rats at 7,14 , and 21 days of age were exposed to unfamiliar adult conspecific males. There are many reports that adult male rats exhibit infanticide (Rosenberg, Denenberg, Zarrow, \& Frank, 1971; Takahashi \& Lore, 1982), and they are more likely to kill young neonates than older weanling rats between the ages of 21 and 25 days (Paul \& Kupferschmidt, 1975). Thus, adult conspecific males appear to represent a significant threat to young preweanling rats. Accordingly, pups at 7 and 14 days old, which are more vulnerable to infanticide than 21-day-old pups, may reduce their emission of ultrasounds when they are near unfamiliar adult males.

\section{Experiment 1}

Conely and Bell (1978) reported a reduction in the emission of ultrasounds when isolated pups were presented with the nest odors of an adult male. They suggested that a reduction in ultrasounds may facilitate survival by reducing the likelihood of detection by adult males. However, Conely and Bell also reported a reduction in ultrasounds when pups were presented with odors from the home cage. On the basis of 
these behavioral data, it is not possible to unequivocally determine whether an unfamiliar adult male represents a significant threat to preweanling rats.

Therefore, the purpose of Experiment 1 was to examine whether preweanling rats selectively reduce their emission of ultrasounds in response to an adult male. Male pups were placed in a compartment adjacent to another that was empty or that contained either an unfamiliar adult male or the mother. The pups were tested in proximity to their mothers because studies have reported that mothers' physical presence is highly effective in reducing pups' propensity to emit ultrasounds (Hofer \& Shair, 1978, 1987). Hence, any reduction in ultrasounds induced by proximity to adult males could be compared with the levels that occurred when pups were in proximity to the mother. Plasma ACTH was measured to facilitate the interpretation of a reduction in ultrasounds in tests with the adult male and with the mother. Secretion of pituitary ACTH provides a physiological measure of the stressfulness or arousal induced by exposure to the different stimulus conditions. Pituitary-adrenal hormone concentrations are reported to increase as a function of stressor intensity (Coover, Sutton, Welle, \& Hart, 1978; Takahashi, Baker, \& Kalin, 1990; Takahashi, Kalin, Barksdale, Vanden Burgt, \& Brownfield, 1988). Thus, it is possible that whereas ultrasound production may be reduced similarly in pups observed near either the male or the mother, ACTH concentrations may only be elevated in tests with the adult male. Such results would suggest that similar behavioral outcomes do not reflect similar physiological processes and that proximity to adult males may be threatening.

\section{Method}

Stimulus animals and subjects. Adult virgin Sprague-Dawley female rats (90-120 days old) were used for breeding. They were offspring, born in this laboratory, of a stock obtained originally from Sasco (Madison, WI). At 21 days of age, the females were housed in isosexual groups of 3-5 animals until 50 days of age. After this age the rats were housed individually in stainless steel hanging cages with access to food and water. A 12:12-hr light:dark photoperiod (lights on at $0600 \mathrm{hr}$ ) was maintained throughout the experiment, and the ambient temperature of the breeding colony was maintained at 22 ${ }^{\circ} \mathrm{C}$. Sexually experienced male Sprague-Dawley rats (150-250 days old) were used for mating and housed under identical conditions. The mothers and several of the sires were used as stimulus animals.

Pairs of male and female rats were kept overnight in breeding boxes, and the next morning a vaginal smear was examined microscopically for the presence of sperm (Day 1 of pregnancy). Mated females were returned to their hanging cages and remained undisturbed, except for weekly cage cleanings, when they were transferred to clean cages. On Day 20 of pregnancy, the females were placed individually in plastic breeding cages $(31 \mathrm{~cm}$ wide $\times 18 \mathrm{~cm}$ high $\times$ $21 \mathrm{~cm}$ deep) with wire-mesh tops. Each cage was provisioned with food, water, and a layer of wood shavings.

Breeding cages were examined daily for the presence of pups (day of birth = postnatal Day 0). Litters were left undisturbed except for routine cage cleaning until the day of the test. A total of 28 litters were produced and assigned to Day $7(n=10)$, Day $14(n=10)$, and Day $21(n=8)$ age groups.

Apparatus. Tests were conducted in a Plexiglas enclosure $(46 \times$ $20 \times 21 \mathrm{~cm}$ ) subdivided into two equal compartments by an alumi- num partition. The enclosure rested on a grid floor elevated $3 \mathrm{~cm}$ above the floor and was housed in a wooden box with an open front for observations. The apparatus was wiped clean after each test.

Test procedure. Although the number of male pups varied in each litter, not more than 4 males were randomly taken from each litter and assigned to one of the four conditions: (a) baseline condition. (b) isolation condition, (c) isolation-unfamiliar male proximity condition, and (d) isolation-maternal proximity condition. A total of 107 rat pups were used. In the baseline condition the pup was removed from the litter and decapitated in an adjacent room in order to determine basal concentrations of plasma ACTH (see later discussion of procedure for measuring $\mathrm{ACTH}$ ). In the isolation condition the pup was placed alone on one side of the two-compartment enclosure, and the adjacent compartment was empty. In the isolationmale proximity condition, the pup was placed alone in one compartment, and a sexually experienced male was placed in the adjacent compartment. In the isolation-maternal proximity condition, the pup was placed alone in one compartment, and its mother was placed in the adjacent compartment. Under these test conditions, auditory and chemosensory stimuli, but not visual or tactile cues, could be transferred between compartments. The ambient temperature of the test enclosure was $22-23{ }^{\circ} \mathrm{C}$. All tests were 10 -min long and were conducted during the first half of the light cycle.

In each test the frequency of occurrence of ultrasonic vocalization was recorded. Ultrasonic vocalizations were detected with an earphone attached to the socket of a minibat detector (QMC Instruments, London) tuned to the appropriate frequencies of $40-50 \mathrm{kHz}$ for 7 - and 14-day-oid rats and $30-40 \mathrm{kHz}$ for 21 -day-old rats (Takahashi, Baker, \& Kalin, 1990). The bat detector transforms the ultrasonic vocalization into the audible range for humans. Each discrete vocalization was counted except those produced during face washing. Ultrasounds made during face washing were not recorded because they appeared to be produced by movements of the forepaws as they were swept forward over the facial vibrissae area. Such ultrasounds may be produced by mechanical properties of face washing and not in response to specific environmental stimuli.

The duration (in seconds) of defensive freezing was also recorded during testing of 21-day-old rats. Freezing was defined as the cessation of body and vibrissae movements except when accompanying respiration. The rat typically assumes the crouching posture when freezing. Because 7- and 14-day-old rats have difficulty assuming a crouching stance, freezing was not measured at these younger ages.

At the conclusion of the $10-\mathrm{min}$ test, pups were decapitated, and trunk blood was collected in ice-chilled tubes of EDTA. Tubes were centrifuged at $4{ }^{\circ} \mathrm{C}$ for $15 \mathrm{~min}$ at $3,000 \mathrm{rpm}$. The plasma was divided into aliquots and stored at $-70^{\circ} \mathrm{C}$ until the time of assay.

Plasma hormone radioimmunoassay (RIA). Plasma ACTH was measured in unextracted plasma in duplicate with a completely characterized antibody raised against $\mathrm{ACTH}_{1-24}$ (IgG, Nashville, TN; Nicholson, Davis, Sherrell, \& Orth, 1984). The minimum known sequence required for complete immunoreactivity with this antiserum is $\mathrm{ACTH}_{5-18}$. The radioactive tracer was obtained from Immunonuclear, Stillwater, MN.) ACTH A $_{1-39}$ (Peninsula Laboratories, Belmont, CA) was used in the standard curve. The sensitivity of the assay was $0.3 \mathrm{pg}$ per tube with an intraassay variability of $4.2 \%$.

Statistics. Data were analyzed with parametric analysis of variance (ANOVA) procedures unless indicated otherwise. Post hoc comparisons were conducted with Tukey's test.

\section{Results}

Ultrasonic vocalizations and freezing. As shown in Figure 1 , a reduction in uitrasonic vocalizations occurred as the rat pups matured. In fact, 21 -day-old pups did not emit ultra- 


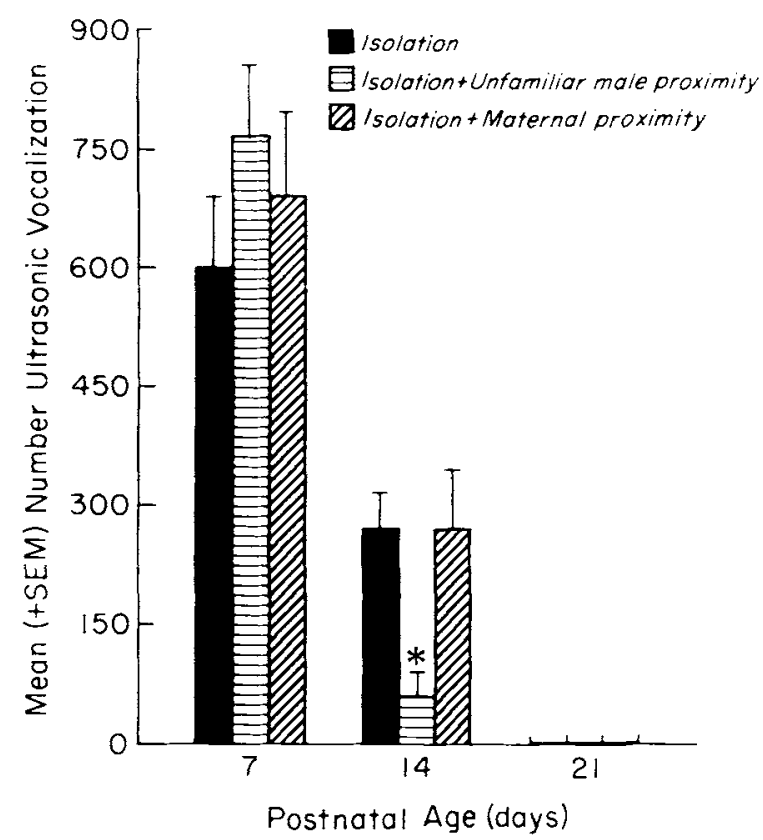

Figure 1. Ultrasonic vocalizations emitted by 7-, 14-, and 21 -dayold male rats during a 10 -min exposure to different stimulus conditions. $\left({ }^{*}\right.$ Significantly different from isolation and maternal proximity groups at $p<.05 ; n \mathbf{s}=8-10$ per group.)

sounds when exposed to any of the three stimulus conditions. The number of pups that emitted ultrasounds when exposed to the various test conditions differed significantly across ages, $\chi^{2}(2, N=81)=82.0, p<.001$. Importantly, in the isolationmale proximity test, 14-day-old pups, but not 7-day-old pups, reduced their production of ultrasounds. A 2 (age) $\times 3$ (stimulus condition) ANOVA conducted on the number of ultrasounds emitted by 7- and 14-day-old pups in the three stimulus conditions indicated a highly significant main effect of age, $F(1,52)=66.18, p<.001$, and a significant interaction, $F(2,52)=5.01, p<.01$. The main effect of stimulus condition was not significant, $F(2,52)=1.25, p>.05$. Exposure to the unfamiliar male was highly effective in reducing the emission of ultrasounds in 14-day-old $(p<.01)$ but not 7-day-old pups (Figure 1). In fact, 7-day-old pups produced similar levels of ultrasounds in all three test conditions.

Two 21-day-old rats exhibited freezing (range, 13-292 s) in the isolation-male proximity test, and one froze (121 s) in the isolation test. No 21 -day-old pup was observed to freeze in the isolation-maternal proximity test.

Plasma $A C T H$. Highly significant main effects in ACTH concentrations were found for age, $F(2,95)=49.52, p<.001$, and stimulus condition, $F(3,95)=15.42, p<.001$. Plasma ACTH increased significantly at each age level $(p<.05)$ and was significantly higher than basal concentrations after exposure to the three test conditions $(p<.01)$. In addition, there was a significant Age $\times$ Stimulus Condition interaction, $F(6,95)=4.50, p<.001$. Plasma ACTH increased significantly in 7-day-old pups in the isolation test $(p<.05)$ but not in the other two test conditions (Figure 2). However, in 14and 21-day-old pups, plasma ACTH was significantly higher than basal concentrations after exposure to the three test conditions $(p<.01)$.

Relations between ultrasonic emission and plasma $A C T H$. To identify potential relations between the number of ultrasounds produced and plasma ACTH concentrations, Pearson product-moment correlation coefficients were computed from data obtained in 7- and 14-day-old rats. Correlational analyses were not conducted for 21 -day-old rats because ultrasounds were not produced.

In rats at 7 days of age, a significant positive relation was found in the isolation-male proximity test $(r=.692, p<.05)$ but not in the isolation test $(r=.529, p>.05)$ or the isolationmaternal proximity test $(r=.460, p>.05)$.

In rats at 14 days of age, a highly significant negative correlation was found in the isolation test $(r=-.822, p<$ .01 ) but not in the isolation-male proximity test $(r=.141, p$ $>.05)$ or the isolation-maternal proximity test $(r=-.311, p$ $>.05)$

\section{Discussion}

The results indicate that at 14 days of age, but not at 7 days, rat pups observed in isolation and in proximity to conspecific adults alter their production of ultrasonic vocalizations. Proximity to an unfamiliar male was highly effective in reducing the emission of ultrasounds, in contrast to tests that involved only isolation or proximity to the mother. This reduced occurrence of ultrasounds in 14-day-old rats when in proximity to an unfamiliar adult male, a putative threat, is similar to previous results that demonstrated that exposure to electric shock significantly reduces the occurrence of ultrasounds (Takahashi, Baker, \& Kalin, 1990).

Unexpectedly, 7- and 14-day-old pups continued to emit ultrasonic vocalizations when in proximity to their mother.

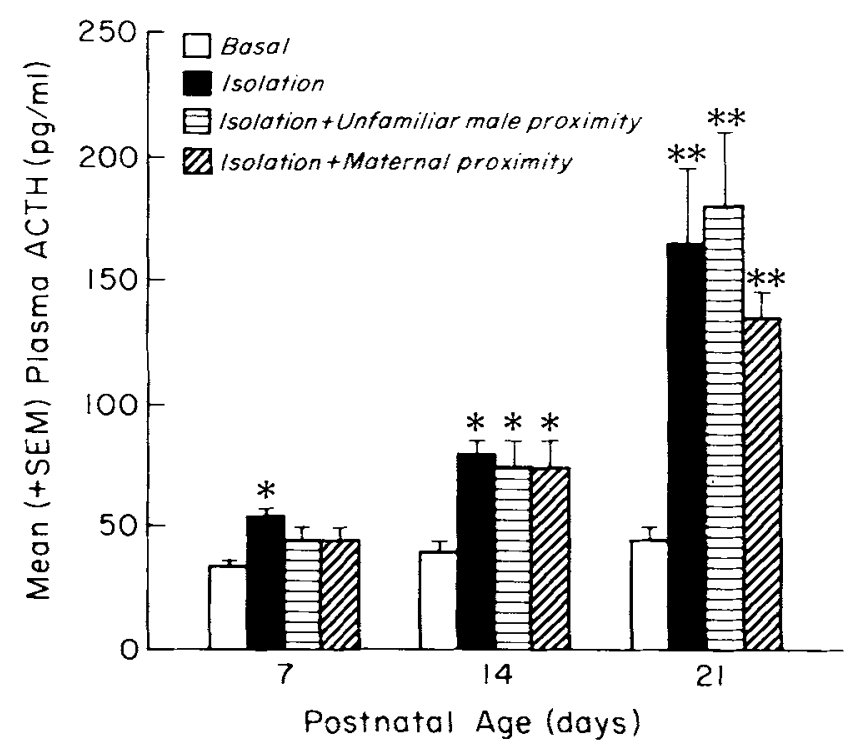

Figure 2. Plasma ACTH concentrations in 7-, 14-, and 21-day-old male rats in the basal state or immediately after a 10-min exposure to different stimulus conditions. (Values for $p$ indicate significant differences from basal concentrations; ${ }^{*} p<.05$, and ${ }^{* *} p<.01$.) 
Although these results appear to conflict with previous reports (Hofer \& Shair, 1978, 1987), it is possible that preweanling rats require physical or tactile stimulation from the mother in order to reduce their production of ultrasounds. In those earlier studies, the reported reduction in ultrasounds occurred in tests in which pups were allowed to engage in physical contact with their mother. In our study, pups were placed in proximity to their mother, but tactile contact was not possible.

Support for the hypothesis that tactile stimulation may ameliorate the effects of unfamiliarity induced by separation from the nest is taken from a study that showed that maternal contact effectively inhibits pituitary-adrenal activation (Stanton, Wallstrom, \& Levine, 1987). Indeed, my data suggest that the absence of physical or tactile stimulation from the mother may be responsible for the increase in plasma ACTH found in 14-day-old rats. At this age, plasma ACTH concentrations, although elevated, did not differ significantly after exposure to either the adult male or the mother. The results suggest that 14-day-old rats observed near, but without tactile stimulation from, their mothers were highly aroused. Their persistence in emitting ultrasounds may serve to direct the mother to their whereabouts.

In 2 I-day-old pups, ultrasonic vocalizations no longer occurred when tested in the three stimulus conditions. However, brief bouts of freezing, a defensive response (Blanchard \& Blanchard, 1969; Ratner, 1967), were occasionally observed in the isolation test and in the isolation-male proximity condition. In addition, 21 -day-old pups in proximity to the male or in isolation tended to have higher ACTH concentrations than did pups in the maternal proximity test. The results may suggest that 21-day-old pups are wary of unfamiliar situations and adult conspecific males.

\section{Experiment 2}

The results of Experiment 1 indicate that 14-day-old rats, but not 7-day-old rats, reduce their emission of ultrasounds when placed in proximity to an adult male. Although this selective reduction in ultrasound production in 14-day-old pups provides the first strong evidence that adult males represent a threat, additional studies with other behavioral measures are necessary in order to fully confirm the hypothesis that unfamiliar adult males represent a significant threat to these pups.

Therefore, Experiment 2 was conducted to characterize further the behavioral responses of preweanling rats when they encounter conspecific adults. Pups at 7, 14, and 21 days old were exposed to the three stimulus conditions used in Experiment 1. However, unlike Experiment 1, a pup was now provided with the opportunity to enter the compartment with the unfamiliar male or with its mother. I hypothesized that if an unfamiliar adult male is a significant threat to preweanling rats, then pups ought to withdraw or avoid entering the stimulus compartment with the male.

\section{Method}

Subjects and apparatus. The subjects were 7-, 14-, and 21 -dayold male Sprague-Dawley rats obtained from litters as described in
Experiment 1. Three male pups were selected randomly from each litter. Seven- and 21 -day-old pups were each derived from 10 litters, and 14-day-old pups were obtained from 14 litters. A total of 102 rat pups were used. Sexually experienced male Sprague-Dawley rats as well as the mothers were used as stimulus animals.

The apparatus described in Experiment I was modified to allow the pup to pass from one compartment to the other. A rectangle $(5.5$ $\mathrm{cm}$ wide $\times 2.0 \mathrm{~cm}$ high) was cut in the bottom center of the aluminum partition. The size of this entry allowed only the pup to freely enter or leave the stimulus compartment. The two-compartment enclosure rested on a cardboard floor that was changed after each test.

Test procedure. Tests were 10 -min in duration and conducted during the first half of the light cycle. Pups were randomly allocated to the different test conditions in the manner indicated in Experiment 1. Responses described in Experiment 1 were recorded as well as the latency (in seconds) to enter the stimulus compartment and the duration (in seconds) spent in the stimulus compartment. Plasma ACTH was not measured in this experiment.

Statistics. Parametric (Winer, 1971) and nonparametric (Siegel, 1956) procedures were used to analyze the data. Parametric analysis was the method of choice when data were normally distributed.

\section{Results}

Latency to enter and time spent in the stimulus compartment. As indicated in Table 1, the proportion of pups that entered the stimulus compartment in each condition increased significantly as the animals matured, $\chi^{2}(2, N=102)$ $=35.4, p<.001$. No significant differences were found in the proportion of 7-day-old pups that entered the other compartment in each condition. In fact, in all three test conditions, 7 -day-old rats rarely entered the stimulus compartment. The proportion of 21-day-old pups that entered the stimulus compartment also did not differ. Unlike 7-day-old pups, however, most 21-day-old pups entered the other compartment. In contrast to 7-and 21-day-old pups, the proportion of 14-dayold pups that entered the other compartment was nonrandomly distributed, $\chi^{2}(2, N=42)=9.9, p<.01$. A significantly

Table 1

Responses of 7-, 14-, and 21-Day-Old Rats When Exposed for I0-Min to Three Different Stimulus Conditions

\begin{tabular}{|c|c|c|c|c|}
\hline & & & Latency & Time \\
\hline Age and condition & $n$ & Proportion & $M \pm S E_{M}$ & $M \pm S E_{M t}$ \\
\hline \multicolumn{5}{|l|}{7 days } \\
\hline Isolation & 10 & 1 & $571 \pm 29$ & $18 \pm 18$ \\
\hline Isolation-male & 10 & 1 & $598 \pm 2$ & $3 \pm 2$ \\
\hline Isolation-mother & 10 & 2 & $497 \pm 69$ & $103 \pm 69$ \\
\hline \multicolumn{5}{|l|}{14 days } \\
\hline Isolation & 14 & $8^{a}$ & $398 \pm 60$ & $103 \pm 40^{\mathrm{a}, \mathrm{b}}$ \\
\hline Isolation-male & 14 & $2^{c}$ & $565 \pm 27^{b}$ & $35 \pm 27^{\circ}$ \\
\hline Isolation-mother & 14 & 10 & $314 \pm 61$ & $284 \pm 61$ \\
\hline \multicolumn{5}{|l|}{21 days } \\
\hline Isolation & 10 & 9 & $266 \pm 81^{b}$ & $161 \pm 51^{\circ}$ \\
\hline Isolation-male & 10 & 8 & $320 \pm 63^{c}$ & $252 \pm 57^{c}$ \\
\hline Isolation-mother & 10 & 10 & $61 \pm 3$ & $459 \pm 16$ \\
\hline
\end{tabular}

Note. Proportion $=$ proportion of pups that entered the stimulus compartment; Latency = latency to enter the stimulus compartment; and Time $=$ time spent in the stimulus compartment.

${ }^{a}$ Significantly different from isolation-male group at $p<.05$. ${ }^{b}$ Significantly different from isolation-mother group at $p<.05$. c Significantly different from isolation-mother group at $p<.01$. 
greater proportion of 14-day-old pups entered either the empty compartment or the compartment with the mother than they did the compartment with an unfamiliar adult male $(p<.05$, Fisher exact probability test).

Subsequent analyses were conducted to examine differences within each age group. Latency data were analyzed in a oneway ANOVA. If the pup did not enter the stimulus compartment, the latency measure was considered to be $600 \mathrm{~s}$. Latency scores in 7-day-old pups did not differ significantly among the three test groups, $F(2,27)=1.45, p>.05$. However, at 14 and 21 days of age, significant group differences emerged in the latency to enter the various stimulus compartments, $F(2,39)=6.10$ and $F(2,27)=5.15$, respectively, $p$ s $<.05$ (see Table 1). Pups at 14 and 21 days old took significantly more time to enter the compartment with the adult male than they did to enter the compartment with the mother $(p s<.01$, Tukey test). Significantly longer latencies to enter the empty compartment in comparison with the latencies to enter the compartment with the mother rat were also found in 21-dayold rats $(p<.05)$.

The amount of time spent in the stimulus compartment also differed significantly among groups at 14 and 21 days of age, Kruskal-Wallis $H(2)=11.76$ and 12.89 , respectively, $p$ s $<.01$, but not at 7 days of age, $H(2)=0.80, p>.05$. Fourteenand 21-day-old pups spent significantly less time in the compartment with a male (see Table 1) than in the compartment with the mother ( $p s<.01$, Mann-Whitney $U$ tests). In addition, 14- and 21 -day-old pups spent more time in the stimulus compartment with the mother than in the empty stimulus compartment $(p<.05)$.

Ultrasonic vocalizations and freezing. Because rat pups varied the amount of time spent in the two compartments, the number of ultrasounds emitted in each compartment was converted to a rate measure. This rate measure was derived by dividing the total number of ultrasounds produced in a compartment by the total amount of time spent in that compartment. Thus, the rate measure reflects the propensity to emit ultrasounds per unit of time spent in each compartment.

As shown in Table 2, in all three stimulus conditions, 7day-old pups produced a high number of ultrasonic vocalizations, whereas 21 -day-old pups were silent. In addition, three 21-day-old pups exhibited freezing ( $M=30 \mathrm{~s})$ in the start compartment when placed in proximity to an unfamiliar male. In the other two test conditions, freezing was not observed.

Analysis on the number of ultrasounds emitted in the start compartment indicated significant differences among groups, $H(2)=14.13, p<.001$. Fourteen-day-old pups observed with the unfamiliar adult male produced fewer ultrasounds than did pups in the other two conditions ( $p<.05$, Mann-Whitney $U$ test). The results replicate data obtained in Experiment 1 that showed that when 14-day-old pups were tested in proximity to adult males, they significantly reduced their production of ultrasounds.

Further analysis indicates that 14-day-old pups that entered the compartment with their mother or the empty compartment significantly reduced their emission of ultrasounds $(p<$ .05 , Wilcoxon signed-ranks test). Too few 14-day-old pups
Table 2

Spatial Distribution Rate of Ultrasonic Vocalizations

Produced by 7-, 14-, and 21-Day-Old Rats During a 10-Min Exposure to Three Different Stimulus Conditions

\begin{tabular}{|c|c|c|c|c|}
\hline \multirow[b]{3}{*}{ Age and condition } & \multicolumn{4}{|c|}{ Ultrasonic vocalizations per minute } \\
\hline & \multicolumn{2}{|c|}{$\begin{array}{c}\text { Start } \\
\text { compartment }\end{array}$} & \multicolumn{2}{|c|}{$\begin{array}{l}\text { Stimulus } \\
\text { compartment }\end{array}$} \\
\hline & $n$ & $M \pm S E_{M}$ & $n$ & $M \pm S E_{M}$ \\
\hline \multicolumn{5}{|l|}{ Day 7} \\
\hline Isolation & 10 & $62 \pm 6$ & 1 & 64 \\
\hline Isolation-male & 10 & $55 \pm 7$ & 1 & 0 \\
\hline Isolation-mother & 10 & $78 \pm 8$ & 2 & $16 \pm 10$ \\
\hline \multicolumn{5}{|l|}{ Day 14} \\
\hline Isolation & 14 & $22 \pm 4$ & 8 & $13 \pm 6^{b}$ \\
\hline Isolation-male & 14 & $6 \pm 3^{a}$ & 2 & $0 \pm 0$ \\
\hline Isolation-mother & 14 & $14 \pm 4$ & 10 & $5 \pm 3^{c}$ \\
\hline \multicolumn{5}{|l|}{ Day 21} \\
\hline Isolation & 10 & $0 \pm 0$ & 9 & $0 \pm 0$ \\
\hline Isolation-male & 10 & $0 \pm 0$ & 8 & $0 \pm 0$ \\
\hline Isolation-mother & 10 & $0 \pm 0$ & 10 & $0 \pm 0$ \\
\hline
\end{tabular}

Note. Means and standard errors are based on the number of vocalizers only, which are given in the column for sample size.

${ }^{2}$ Significantly different from isolation and isolation-mother groups at $p<.05$. ${ }^{\mathrm{b}}$ Significantly different from number of ultrasounds emitted in start compartment at $p<.05$. ' Significantly different from number of ultrasounds emitted in start compartment at $p<$ .01 .

entered the compartment with the male to provide meaningful comparisons in ultrasonic production in the two compartments. However, it is notable that in 2 cases, contact with the male elicited immobility or a freezelike condition. These 14 day-old pups remained motionless as they lay on the floor of the compartment. Observations of the behavior of the adult males toward the pups indicated that the males initially spent a great amount of time sniffing the pups. Subsequently, they appeared to ignore their presence. No adult male was observed to attack a pup.

\section{Discussion}

The results of Experiment 2 show that 14-day-old rats avoided the compartment with the unfamiliar adult male and reduced their production of ultrasonic vocalizations. This withdrawal or avoidance response, which is frequently exhibited when organisms sense danger (Barnett \& Spencer, 1951; Metzgar, 1967; Schaller, 1972; Takahashi, Grossfeld, \& Lore, 1980; Takahashi, Kalin, \& Baker, 1990), is considered to be a behavioral hallmark of fear (Hinde, 1970). The results provide clear evidence that (a) an unfamiliar male was a significant threat to 14-day-old rats and (b) 14-day-old rats responded to this threat by reducing their emission of ultrasounds.

The results further show that the propensity to withdraw from an unfamiliar adult male declines with age. That is, although 21-day-old pups appear to be wary of initiating interactions with adult males, as indicated by high latencies to enter the male's compartment, the proportion of pups that eventually entered the compartment with the male did not differ from the proportion that entered the compartment with their mother. 
Although few 7-day-old pups entered the other compartment in any of the three conditions, this result must not be interpreted as evidence for a predisposition of young and still blind rat pups to avoid conspecific adults. At 7 days old, pups have not yet developed strong locomotor responses. Consequently, in Experiment 2, the 7-day-old rats were not able to exploit fully the opportunity to interact with the mother, which required the pup to locate the passageway and enter the adjacent compartment. What appears to be important, however, is that 7-day-old pups, unlike older 14-day-old pups, persisted in their emission of ultrasounds when tested in all three stimulus conditions.

\section{Experiment 3}

Experiments 1 and 2 indicated that 7-day-old rats did not selectively reduce their production of ultrasonic vocalizations when observed in proximity to adult males. Instead, ultrasound production was similar across the three stimulus conditions. An interpretation of these data is that 7-day-old pups, unlike older 14-day-old pups, may not able to differentiate the varied stimulus conditions. However, in Experiment 1, plasma ACTH in 7-day-old rats increased significantly from basal concentrations in the isolation test, and there was a significant positive correlation between ultrasound production and plasma ACTH concentrations in the male proximity test. This result underscores the importance of measuring plasma ACTH because it suggests that 7-day-old pups were able to differentiate the stimulus conditions, albeit this differentiation was not reflected by an alteration in ultrasound production.

Therefore, the purpose of Experiment 3 was to examine further whether 7-day-old rats are capable of altering their behavioral responses when tested with conspecific adults. On the basis of a very limited sample size, 7-day-old pups that entered the compartment with either their mother or the adult male reduced their emission of ultrasounds (Experiment 2, Table 2). Whether this reduction in ultrasounds differs significantly in 7-day-old pups observed in direct proximity to an unfamiliar male and the mother, however, remains to be determined. Therefore, 7-day-old pups were placed in a compartment either with their mother or with an unfamiliar adult male. To eliminate the possibility that differences among groups result from differences in adult behavior toward the pups, the adult rats were anesthetized before observations were made. This experiment differs from Experiment $I$ in that conspecific tactile stimuli were present. As in Experiment 1, plasma ACTH was measured in order to provide another basis for assessing whether 7-day-old pups are capable of differentiating the varied stimulus conditions.

\section{Method}

Subjects and apparatus. Thirty-three 7-day-old male SpragueDawley rats were obtained from litters $(n=10)$ as described in Experiment 1. Sexually experienced male Sprague-Dawley rats as well as the mothers were used as stimulus animals.

Tests were conducted in one of the sealed compartments of the apparatus described in Experiment 1. The apparatus rested on a cardboard floor that was changed after each test, and the interior of the compartment was wiped with ethanol.

Test procedure. Three 7-day-old male rats were randomly selected from each litter and tested in one of the three stimulus conditions, (a) social isolation, (b) exposure to an anesthetized male, or (c) exposure to an anesthetized mother. An additional male pup from each litter served as a baseline control as described in Experiment 1.

Adult stimulus rats were anesthetized with sodium pentobarbital $(50 \mathrm{mg} / \mathrm{kg}$ ip). Mother rats were removed from the nest box immediately before the litter was tested, and after injection of sodium pentobarbital, they were placed in a clean holding cage. Anesthetized adults were placed in the compartment on their ventral surface to prevent the pups observed with the mother from sucking its nipples.

The number of ultrasonic vocalizations emitted was recorded as described in Experiment 1. At the conclusion of behavioral testing, pups were decapitated, trunk blood collected, and plasma stored as described previously. ACTH was measured with RIA procedures described in Experiment 1.

Statistics. Data were analyzed in one-way ANOVAs to establish overall significance. Post hoc comparisons were made with Tukey's test. Pearson product-moment correlation coefficients between the number of ultrasounds emitted and plasma ACTH concentrations were also computed.

\section{Results}

Ultrasonic vocalization. Significant group differences were found in ultrasonic vocalization, $F(2,23)=8.04, p<$ .01 (Figure 3). Seven-day-old pups observed in either the anesthetized male or the anesthetized mother condition emitted significantly fewer ultrasounds than pups observed in isolation $(p s<.01)$. Although the amount of time in physical contact with the anesthetized adult was not measured, all pups approached and contacted the adult.

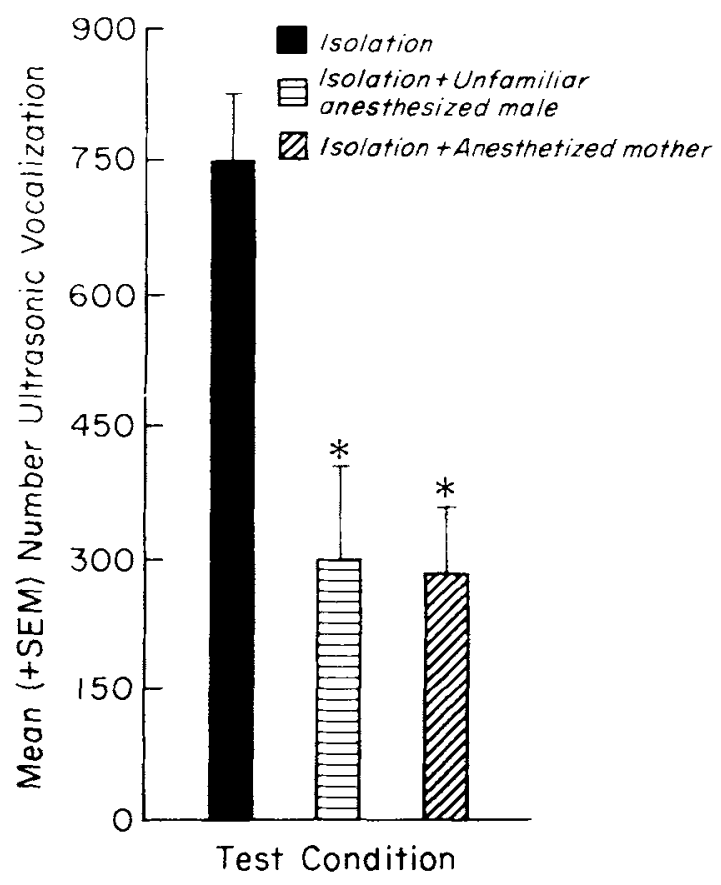

Figure 3. Ultrasonic vocalizations emitted by 7-day-old male rats during exposure to different stimulus conditions. ( ${ }^{*}$ Significantly different from isolation group at $p<.01 ; n \mathrm{~s}=7-10$ per group.) 
Plasma ACTH. ACTH concentrations differed significantly among groups, $F(3,29)=12.04, p<.001$. Pups in the isolation test as well as in the anesthetized male test had significantly higher plasma ACTH levels than pups in the anesthetized mother test ( $p s<.01$; Figure 4$)$. In fact, 7-dayold pups in the anesthetized mother test had plasma ACTH concentrations that did not differ significantly from basal levels. Olfactory recognition (Hepper, 1986; Schapiro \& Salas, 1970) in conjunction with maternal tactile stimuli (Hofer \& Shair, 1978, 1987) appear to be effective in reducing not only the production of ultrasonic vocalizations but also the secretion of pituitary ACTH.

Relations between behavioral and hormonal responses. There was a significant positive correlation between ultrasound emission and plasma $\mathrm{ACTH}$ in 7-day-old pups tested with the anesthetized male $(r=.710, p<.05)$. No significant correlations, however, were found in either the isolation test $(r=.327, p>.05)$ or the anesthetized mother test $(r=.276, p>.05)$.

\section{Discussion}

The key question in this experiment is based on stimulus and motivational considerations: Do 7-day-old pups perceive the adult male as a threat? As shown in Experiment 1, 7-dayold pups, unlike older 14-day-old rats, did not reduce their ultrasonic vocalizations in the male proximity test. In addition, results of Experiment 3 indicate that although 7-day-old pups reduced their output of ultrasounds in the anesthetized male test, no significant differences in the production of ultrasounds were found between pups in the anesthetized

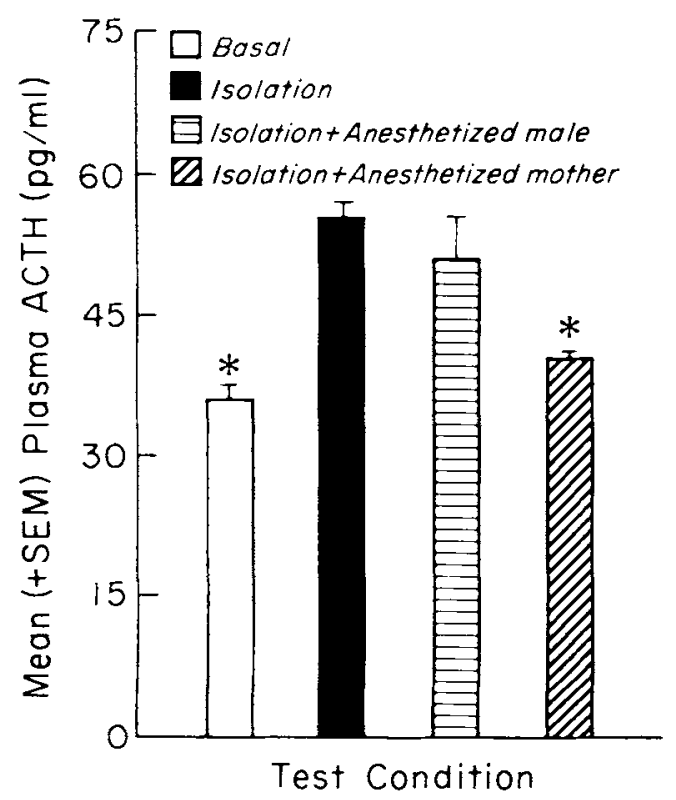

Figure 4. Plasma ACTH concentrations in 7-day-old male rats in the basal state or immediately after a 10 -min exposure to different stimulus conditions. (*Significantly different from isolation and anesthetized male groups at $p<.01 ; n s=7-10$ per group.) male and the anesthetized mother tests. Seven-day-old pups, however, appeared capable of distinguishing the mother from the adult male, as evidenced by the significant group differences in plasma ACTH concentrations and the positive correlation between behavioral and hormonal measures taken in the anesthetized male test. Further studies will be required to determine whether the significant elevation in plasma $\mathrm{ACTH}$ induced in the anesthetized male test is a result of mere unfamiliarity or a response to threat. For example, threatinduced responses ought to habituate more slowly than responses induced by unfamiliar but nonthreatening stimuli (Magurran \& Girling, 1986).

\section{General Discussion}

These experiments provide data on the development of the preweanling rat's ability to modulate its expression of ultrasonic vocalization in response to conspecific adults. The results suggest that although unfamiliar conspecific adult males may pose a significant threat to 14-day-old pups, by 21 days of age, pups no longer appear to be significantly threatened by their presence. Thus, between the 2 nd and 3rd weeks of postnatal life, rat pups exhibit a significant change in their responses toward unfamiliar adult conspecific males.

At 7 days of age, the rat pup responds to social isolation and unfamiliar situations by emitting a high number of ultrasounds. These vocalizations may induce maternal retrieval behavior, which may be one of the most effective strategies by which young isolated preweanling rats are able to regain contact with the mother. There are several reasons to suggest why 7-day-old pups did not reduce their emission of ultrasounds in response to an unfamiliar adult male. First, the limited locomotor ability of 7-day-old pups presumably restricts their movements to the confines of the nest. Furthermore, because 7-day-old pups are unable to thermoregulate (Adolph, 1957; Okon, 1971; Taylor, 1960), the likelihood of their leaving the nest is low. Under these conditions, distant separation from the nest may be a rare event. Bolles and Woods (1964) observed that during the 1st week of life, pups generally became isolated when the mother left the nest and in the process dragged nursing pups with her. Thus, because separation is limited spatially to the immediate vicinity of the nest, which the mother can defend (Wolff, 1985), and maternal defense in rodents is reported to be elevated during the Ist postpartum week (Flannelly, Flannelly, \& Lore, 1986; Gandelman, 1972; Svare, Betteridge, Katz, \& Samuels, 1981), there may not be strong selective pressures acting on very young preweanling Norway rats to inhibit their emission of ultrasounds when exposed to threatening stimuli. Another explanation is that during testing, 7-day-old pups suffered a significant reduction in body temperature that activated metabolic heat production and consequent oxygen consumption. This increased delivery of oxygen may stimulate the production of ultrasounds as the by-product of an increase in respiration (Blumberg \& Alberts, 1990). Accordingly, because hairless 7 -day-old pups have a larger surface-to-volume ratio than 14-day-old rats, a greater metabolic demand and consequent increase in the emission of ultrasounds is induced by isolation and thereby masks any potential tendency in 7-day- 
old pups to reduce their emission of ultrasounds when exposed to a threat.

At 14 days of age, locomotor development has increased, and rats pups may wander further from the nest (Rosenblatt \& Lehrman, 1963). These excursions from the nest, however, may also increase the likelihood of getting lost and encountering danger in the absence of maternal protection. My experiments indicate that 14-day-old rats have developed the capacity to modulate or reduce ultrasonic vocalization and withdraw when they encounter unfamiliar conspecific adult males. Young of other species that emit calls are also reported to reduce their vocalizations and inhibit their activity when alarmed (Miller, 1980).

The reduced occurrence of ultrasonic vocalizations in threatened 14-day-old rats has implications for understanding the motivational state of the animal at this age. Previous studies suggested that ultrasounds are emitted when pups are in a state of distress or discomfort (Hofer \& Shair, 1978; Kehoe \& Blass, 1986; Zippelius \& Schleidt, 1956). Accordingly, a decrease in the production of ultrasounds may be interpreted to reflect a decreased state of distress or arousal (Bell, 1974). However, my results as well as those in which a reduction in uitrasounds after exposure to electric shock has been reported (Takahashi, Baker, \& Kalin, 1990) indicate that under some conditions a reduction in ultrasounds also occurs when the pup is threatened during social isolation. Further support for the view that ultrasound production in 14-dayold rats may not always be a reflection of distress or arousal is taken from the hormonal data obtained in Experiment 1, which showed that in the isolation test the plasma ACTH concentrations were negatively correlated with emission of ultrasounds.

The results obtained in 14-day-old rats are similar, in part, to research conducted in nonhuman primates. Infant rhesus monkeys are reported to increase their rate of vocalizations with or without physical contact with their mother but show no increase in plasma cortisol (Bayart, Hayashi, Faull, Barchas, \& Levine, 1990; Levine, Johnson, \& Gonzalez, 1985). However, when placed in total social isolation, infant rhesus monkeys produced few vocalizations but showed a significant increase in plasma cortisol. The researchers suggested that infant rhesus monkeys produce vocalizations in order to facilitate maternal proximity, which is highly effective in reducing arousal. My experiments as well as others conducted in other laboratories (Hennessy \& Moorman, 1989; Hofer \& Shair, 1978, 1987; Stanton et al., 1987) have indicated that in rodents, physical contact with the mother also appears to be effective in reducing secretion of pituitary-adrenal hormones and vocalizations, as compared with the effects of social isolation. However, unlike the infant rhesus monkey, proximity to the mother rat without tactile contact is not less physiologically arousing than social isolation.

Finally, at 21 days of age, social isolation is no longer effective in eliciting ultrasonic vocalizations. In addition, although 21 -day-old pups were initially wary of unfamiliar adult males as shown in Experiment 2, most eventually entered the compartment with the male. Hepper (1986) also reported that rat pups between 18 and 22 days of age began to exhibit a preference for proximity to an unfamiliar conspecific adult male. Importantly, these ages coincide with the time when the propensity of adult males to kill pups decreases significantly (Paul \& Kupferschmidt, 1975). This reduced tendency of adult males to kill pups may provide the impetus for older preweanling pups to interact and familiarize themselves with other group members.

\section{References}

Adolph, E. F. (1957). Ontogeny of physiological regulations in the rat. Quarterly Review of Biology, 32, 89-137.

Allin, J. T., \& Banks, E. M. (1972). Functional aspects of ultrasound production in infant albino rats (Rattus norvegicus). Animal Behaviour, 20, 175-185.

Altman, J., \& Sudarshan, K. (1975). Postnatal development and locomotion in the laboratory rat. Animal Behaviour, 20, 896-920.

Barnett, S. A., \& Spencer, M. M. (1951). Feeding, social behaviour and interspecific competition in wild rats. Behaviour, 3, 229-242.

Bayart, F., Hayashi, K. T., Faull, K. F., Barchas, J. D., \& Levine, S. (1990). Influence of maternal proximity on behavioral and physiological responses to separation in infant thesus monkeys ( $M a c a c a$ mulatta). Behavioral Neuroscience, 104, 98-107.

Bell, R. W. (1974). Ultrasounds in small rodents: Arousal-produced and arousal-producing. Developmental Psychobiology, 7, 39-42.

Blanchard, R. J., \& Blanchard, D. C. (1969). Crouching as an index of fear. Journal of Comparative and Physiological Psychology, 67, 370-375.

Blumberg, M. S., \& Alberts, J. R. (1990). Ultrasonic vocalizations by rat pups in the cold: An acoustic by-product of laryngeal braking? Behavioral Neuroscience, 104, 808-817.

Bolles, R. C., \& Woods, P. J. (1964). The ontogeny of behaviour in the albino rat. Animal Behaviour, 12, 427-444.

Conely, L., \& Bell, R. W. (1978). Neonatal ultrasounds elicited by odor cues. Developmental Psychobiology, 11, 193-197.

Coover, G. D., Sutton, B. R., Welle, S. L., \& Hart, R. P. (1978). Corticosterone responses, hurdle-jump acquisition, and the effects of dexamethasone using classical conditioning of fear. Hormones and Behavior, 11, 279-294.

De Ghett, V. J. (1974). Developmental changes in the rate of ultrasonic vocalization in the Mongolian gerbil. Developmental Psychobiology, 7, 267-272.

Flannelly, K. J., Flannelly, L., \& Lore, R. (1986). Post partum aggression against intruding male conspecifics in Sprague-Dawley rats. Behavioural Processes, 13, 279-286.

Gandelman, R. (1972). Mice: Postpartum aggression elicited by the presence of an intruder. Hormones and Behavior, 3, 23-28.

Hart, F. M., \& King, J. A. (1966). Distress vocalizations of young in two subspecies of Peromyscus maniculatus. Journal of Mammalogy, 47, 287-293.

Hennessy, M. B., \& Moorman, L. (1989). Factors influencing cortisol and behavioral responses to maternal separation in guinea pigs. Behavioral Neuroscience, 103, 378-385.

Hepper, P. G. (1986). Parental recognition in the rat. The Quarterly Journal of Experimental Psychology, 38B, 151-160.

Hinde, R. A. (1970). Animal behaviour. New York: McGraw-Hill.

Hofer, M. A., \& Shair, H. (1978). Ultrasonic vocalization during social interaction and isolation in 2-week-old rats. Developmental Psychobiology, 11, 495-504.

Hofer, M. A., \& Shair, H. (1987). Isolation distress in two-week-old rats: Influence of home cage, social companions, and prior experience with lifter mates. Developmental Psychobiology, 20, 465-476.

Kehoe, P., \& Blass, E. M. (1986). Opioid-mediation of separation distress in 10-day-old rats: Reversal of stress by maternal stimuli. Developmental Psychobiology, 19, 385-398. 
Levine, S., Johnson, D. F., \& Gonzalez, C. A. (1985). Behavioral and hormonal responses to separation in infant rhesus monkeys and mothers. Behavioral Neuroscience, 99, 399-410.

Magurran, A. E., \& Girling, S. L. (1986). Predator model recognition and response habituation in shoaling minnows. Animal Behaviour, $34,510-518$.

Metzgar, L. H. (1967). An experimental comparison of screech owl predation on resident and transient white-footed mice (Peromyscus leucopus). Journal of Mammalogy, 48, 387-391.

Miller, D. B. (1980). Maternal vocal control of behavioral inhibition in mallard ducklings (Anas platyrhynchos). Journal of Comparative and Physiological Psychology, 94, 606-623.

Nicholson, W. E., Davis, D. R., Sherrell, B. J., \& Orth, D. N. (1984). Rapid radioimmunoassay for corticotropin in unextracted human plasma. Clinical Chemistry, 30, 259-269.

Noirot, E. (1966). Ultra-sounds in young rodents: I. Changes with age in albino mice. Animal Behaviour, 14, 459-462.

Noirot, E. (1968). Ultrasounds in young rodents: II. Changes in age in albino rats. Animal Behaviour, 16, 129-134.

Noirot, E. (1972). Ultrasounds and maternal behavior in small rodents. Developmental Psychobiology, 5, 371-387.

Okon, E. (1971). The temperature relations of vocalization in infant golden hamsters and Wistar rats. Journal of Zoology, 164, 227237.

Paul, L., \& Kupferschmidt, J. (1975). Killing of conspecific and mouse young by male rats. Journal of Comparative and Physiolog ical Psychology, 88, 755-763.

Ratner, S. C. (1967). Comparative aspects of hypnosis. In J. E. Gordon (Ed.), Hondbook of clinical and experimental hypnosis (pp. 550-587). New York: Macmillan

Rosenberg, K. M., Denenberg, V. H., Zarrow, M. X., \& Frank, B. L. (1971). Effects of neonatal castration and testosterone on the rat's pup killing behavior and activity. Physiology and Behavior, 7, 363368.

Rosenblatt, J. S., \& Lehrman, D. S. (1963). Maternal behavior of the laboratory rat. In H. L. Rheingold (Ed.), Maternal behavior in mammals (pp. 8-57). New York: Wiley.

Schaller, G. B. (1972). The Serengeti lion. Chicago: University of Chicago Press.

Schapiro, S., \& Salas, M. (1970). Behavioral responses of infant rats to maternal odor. Physiology and Behavior, 5, 815-817.

Siegel, S. (1956). Nonparametric statistics for the behavioral sciences. New York: McGraw-Hill.
Smotherman, W. P., Bell, R. W., Starzec, J., Elias, J., \& Zachman, T. A. (1974). Maternal responses to infant vocalizations and olfactory cues in rats and mice. Behavioral Biology, 12, 55-66.

Stanton, M. E., Wallstrom, J., \& Levine, S. (1987). Maternal contact inhibits pituitary-adrenal stress responses in preweanling rats. $D e-$ velopmental Psychobiology, 20, 131-145.

Svare, B., Betteridge, C., Katz, D., \& Samuels, O. (1981). Some situational and experiential determinants of maternal aggression in mice. Physiology and Behavior, 26, 253-258.

Takahashi, L. K., Baker, E. W., \& Kalin, N. H. (1990). Ontogeny of behavioral and hormonal responses to stress in prenatally stressed male rat pups. Physiology and Behavior, 47, 357-364.

Takahashi, L. K., Grossfeld, S., \& Lore, R. K. (1980). Attack and escape in the laboratory rat: A modification of the colony-intruder procedure. Behavioral and Neural Biology, 29, 512-517.

Takahashi, L. K., Kalin, N. H., \& Baker, E. W. (1990). Corticotropinreleasing factor antagonist attenuates defensive-withdrawal behavior elicited by odors of stressed conspecifics. Behavioral Neurosci ence, 104, 386-389.

Takahashi, L. K., Kalin, N. H., Barksdale, C. M., Vanden Burgt, J. A., \& Brownfield, M. S. (1988). Stressor controllability during pregnancy influences pituitary-adrenal hormone concentrations and analgesic responsiveness in offspring. Physiology and Behavior, 42, 323-329.

Takahashi, L. K., \& Lore, R. (1982). Intermale and maternal aggression in adult rats tested at different ages. Physiology and Behavior, 29, 1013-1018.

Taylor, P. M. (1960). Oxygen consumption in new-born rats. Journal of Physiology, 154, 153-168.

Winer, B. J. (1971). Statistical principles in experimental design (2nd ed.). New York: McGraw-Hill.

Wolff, J. O. (1985). Maternal aggression as a deterrent to infanticide in Peromyscus leucopus and P. maniculatus. Animal Behaviour 33, 117-123.

Zippelius, H. M., \& Schleidt, W. M. (1956). Ultraschallaute bei jungen Mausen [Ultrasounds in young mice]. Naturwissenschaften, 43 , 508.

Received March 29, 1991

Revision received June 5, 1991

Accepted June 11, 1991

\section{Zahn-Waxler Appointed New Editor, 1993-1998}

The Publications and Communications Board of the American Psychological Association announces the appointment of Carolyn Zahn-Waxler as editor of Developmental Psychology. Zahn-Waxler is associated with the National Institute of Mental Health. As of January I, 1992, manuscripts should be directed to

Carolyn Zahn-Waxler
4305 Dresden Street
Kensington, Maryland 20895

Manuscript submission patterns make the precise date of completion of the 1992 volume uncertain. The current editor will receive and consider manuscripts through December 1991. Should the 1992 volume be completed before that date, manuscripts will be redirected to the incoming editor for consideration in the 1993 volume. 